\title{
Minimum Curve Radii for High-Speed Trains, Including the Gyroscopic Moments of the Wheels
}

\author{
Ronald L. Huston \\ Emeritus of Mechanics Mechanical and Materials Engineering University of Cincinnati, Cincinnati, OH, USA \\ Email: ron.huston@uc.edu
}

How to cite this paper: Huston, R.L. (2017) Minimum Curve Radii for High-Speed Trains, Including the Gyroscopic Moments of the Wheels. World Journal of Engineering and Technology, 5, 113-124. https://doi.org/10.4236/wjet.2017.51010

Received: January 5, 2017

Accepted: February 25, 2017

Published: February 28, 2017

Copyright $\odot 2017$ by author and Scientific Research Publishing Inc. This work is licensed under the Creative Commons Attribution International License (CC BY 4.0).

http://creativecommons.org/licenses/by/4.0/

\section{(c) (7) Open Access}

\begin{abstract}
This paper studies the title problem including an analysis of the gyroscopic effects of the wheels of a rail-car travelling at high-speed around a level, horizontal curve. The analysis is based upon the fundamental principles of dynamics. The result is a design formula for the minimum curve radius needed to prevent derailment. Aside from the rail car geometric and physical properties, the minimum curve radius depends upon the square the train speed. An illustrative example shows that the wheel gyroscopic effect is destabilizing and additive to the centrifugal force derailment tendency. From a track design perspective, however, the gyroscopic effect is relatively small compared with the centrifugal force effect.
\end{abstract}

\section{Keywords}

High-Speed Train, Rail Track Design, Wheel Gyroscopic Moments

\section{Introduction}

Recently there has been increased interest in the development of high-speed passenger trains-going at speeds of $483 \mathrm{~km} / \mathrm{hr}$ (300 mph). These trains are envisioned to have numerous advantages over other means of travel:

1) High-speed rail provides rapid personnel transit between the "downtown" areas of major cities.

2) Unlike air travel, with its delays in boarding, takeoff, landing, and deplaning, high-speed rail either reduces or eliminates each of these delays.

3) Also, unlike air travel, high-speed rail is less susceptible to weather delays and/or cancellations.

4) High-speed rail stations are in the city centers whereas airports are usually many kilometers away, requiring time-consuming ground transportation. 
5) Compared with motor vehicle traffic, high-speed rail is much faster and traffic jams, road closures, and detours are avoided.

6) Finally, high-speed rail can be powered electrically-thus reducing the carbon emissions associated with airplanes and motor vehicles.

The challenges and issues for high-speed rail, however, are equally numerous:

1) There is the possibility of derailment, producing a crash with loss of life of the same order as an airplane crash.

2) The rail-car wheels and the track geometry need to be continually monitored and maintained meeting high-precision standards.

3) The trains will require reliable computer speed control.

4) For safety and efficiency, insofar as possible, rail curves need to be eliminated. This in turn may require extensive and expensive changes in land topography.

5) The costs of equipment, construction, and maintenance may be prohibitive for most locales.

Since curves cannot be completely eliminated, in this paper we develop an expression for the minimum curve radius needed to prevent derailment in a level horizontal curve. We include the gyroscopic effects developed by the rapidly turning wheels. Unfortunately, these effects increase the derailment tendency of the centrifugal force of the train in the curve.

To intuitively see this, the "law of gyroscopes" was recalled [1] [2].

If a spinning disk (or wheel) is made to turn about an axis different from its axis of rotation, the wheel will attempt to align its own rotation axis with the imposed rotation axis.

Recently, this writer and others have applied the law of gyroscopes with motorcycle dynamics, where wheel gyroscopic effects are paramount.

To apply this rule, with the wheels of a rail-car traveling around a curve to the left as represented in Figure 1, let $X, Y, Z$ be a dextral axis system with origin at

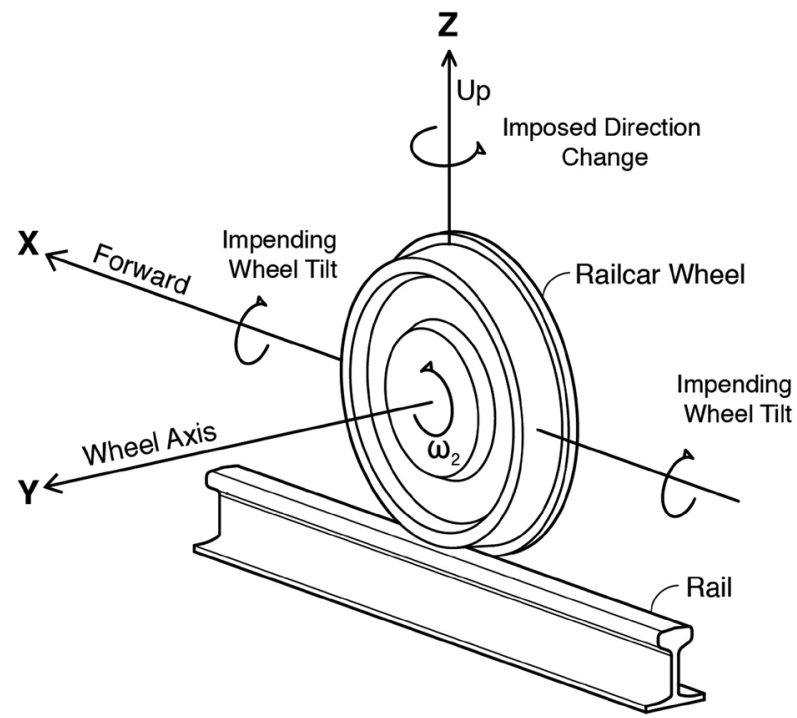

Figure 1. Gyroscopic effect: The wheel axis tends to align with the vertical. 
the wheel center as shown. By inspection of Figure 1 we see that $X$ is forward, $Y$ is the wheel rotation axis, and $Z$ is up. As the train and rail-car are going around a curve to the left, the wheels of the rail-car are forced to rotate about the $Z$-axis. From the law of gyroscopes this imposed rotation causes the wheels to react by attempting to turn about the $X$ axis; thus reinforcing the centrifugal force on the rail-car caused by the train turning to the left.

The balance of the paper is divided into six sections with the first of those providing a brief review of the applicable dynamics equations. In the next section we use these equations to quantify the response of a typical rail-car wheel as the train goes around a curve. In the subsequent section (Section 4) we use the results of the previous analysis to determine the gyroscopic moments produced by the wheels. Finally, in Section 5 we establish the rail-car dynamical equations including design expressions for the minimum safe curve radius. The last two sections present three illustrative computations together with analyses and concluding remarks.

\section{Applicable Dynamics Equations-A Brief Review}

The dynamics of rigid bodies, and even of sets of rigid bodies has been well understood for many years. The theories and analyses are based upon Newton's second law, stating that for a particle $P$ with mass $m$ subjected to a force $F$, the acceleration $a$ of $P$ is

$$
a=F / m \text { or } F=m a
$$

where $a$ is measured in an "inertial" or "fixed" reference frame-a so-called "Newtonian" reference frame. It has also been said that a Newtonian reference frame is a reference frame where Newton's laws are valid.

For practical purposes, and to a high degree of accuracy, in mechanical design, we can consider the earth as a Newtonian reference frame. Using this assumption, the laws of dynamics have been documented in many text books for over 50 years. Our objective is to apply these laws in studying the dynamics of highspeed rail-car wheels.

In our analyses we will use the notation and expressions documented by Kane, et al. [3] [4] [5] [6].

For computational purposes it is convenient to introduce the concept of "inertia forces" (as originally proposed by Rene d'Alembert [3] [4]. The concept is simple: Equation (1) is rewritten in the form:

$$
F+F^{*}=0
$$

where $F^{*}$ is the d'Alembert inertia force defined as:

$$
F=-m a
$$

While the notion of inertia forces is regarded by many analysts as trivial and even illegitimate [7] [8] [9] for our purposes it enables us to quantify the law of gyroscopes and thereby determine the gyroscopic moments of the high-speed rail-car wheels. 
To this end, consider a rigid body $B$ (later to be a rail-car wheel) moving in a Newtonian reference frame $R^{*}$ as represented in Figure 2, where $G$ is the mass center of $B$.

With $B$ being a rigid body, an elementary dynamic analysis shows that the totality of the inertia forces on the particles making up $B$ is equivalent to a single force $F^{*}$, passing through $G$ together with a couple with torque $T^{*}$, where $F^{*}$ and $T^{*}$ may be expressed as [3] [4] [5] [6]:

$$
F^{*}=-M a^{G}
$$

and

$$
T^{*}=-I \cdot \alpha+\omega \times(I \cdot \omega)
$$

where $M$ is the mass of $B, a^{G}$ is the acceleration of $G$ in $R^{*}, I^{*}$ is the inertia dyadic of $B$ relative to

$G$, and finally $\omega$ and $\alpha$ are the angular velocity and angular acceleration of $B$ in $R^{*}$.

Next, let $n_{1}, n_{2}$, and $n_{3}$ be a dextral set of mutually perpendicular unit vectors parallel to the principal inertia directions of $I$ for $G$, as represented in Figure 3, which now also includes the inertia force $F^{*}$ and the inertia torque $T^{*}$.

Let $\omega, \alpha, I$, and $T^{*}$ be expressed in terms of the unit vectors $n_{i}(i=1,2,3)$ as:

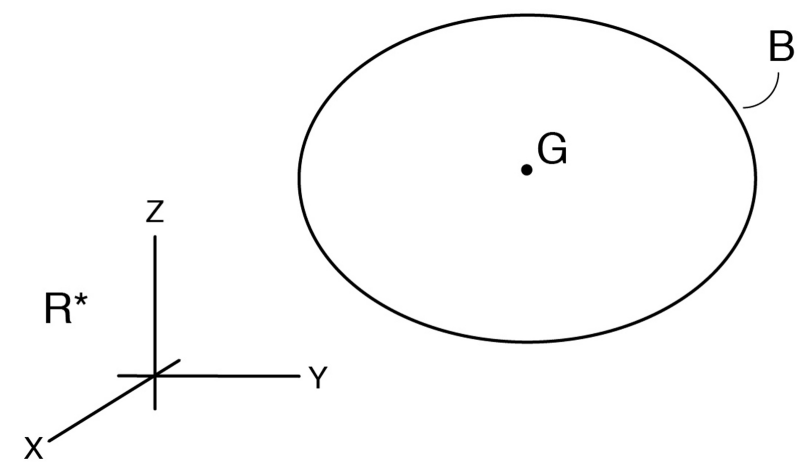

Figure 2. A rigid body $B$ moving in a Newtonian reference frame $R^{*}$.

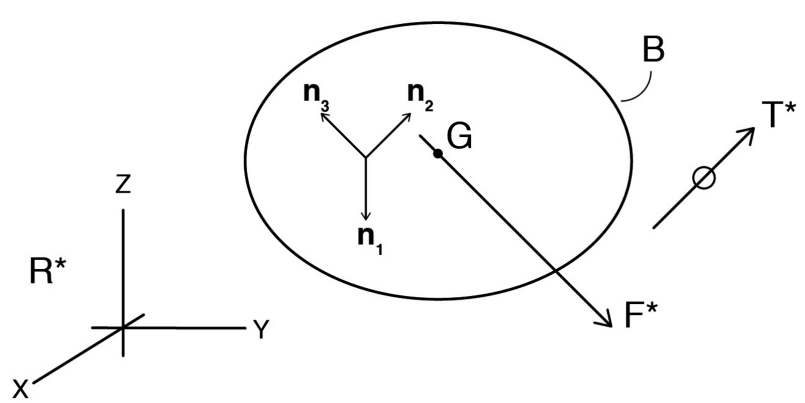

Figure 3. Principal unit vectors $n_{i}(i=1,2,3)$ together with inertia force $F^{*}$ and inertia torque $T^{*}$. 


$$
\begin{gathered}
\omega=\omega_{1} n_{1}+\omega_{2} n_{2}+\omega_{3} n_{3}=\omega_{i} n_{i} \\
\alpha=\alpha_{i} n_{i}, \quad I=I_{i j} n_{i} n_{j}, \quad T^{*}=T_{i} n_{i}
\end{gathered}
$$

where in Equation (6) we introduce the repeated-index-summation convention, and then use it in Equation (7). (Note that with the $n_{i}$ being parallel to principal inertia directions the $I_{i j}, i \pm j$, ("products of inertia") are zero.

By substituting from Equations (6) and (7) into (5) we then obtain the inertia torque components in the forms:

$$
\begin{aligned}
& T_{1}=-\alpha_{1} I_{11}+\omega_{2} \omega_{3}\left(I_{22}-I_{33}\right) \\
& T_{2}=-\alpha_{2} I_{22}+\omega_{3} \omega_{1}\left(I_{33}-I_{11}\right) \\
& T_{3}=-\alpha_{3} I_{33}+\omega_{1} \omega_{2}\left(I_{11}-I_{22}\right)
\end{aligned}
$$

\section{Application with Rail-Car Wheel Dynamics}

Consider a typical rail-car of a high-speed train going around a level horizontal curve with a speed $V$ as in Figure 4. Let the portion of the curve where the rail-car is located be approximated as an arch of a circle with radius $R$.

Next, consider an overhead view of a typical car $C$ of the train as in Figure 5. Let the unit vectors be fixed relative to the car, with $n_{1}$ pointing forward (in the direction of travel), $n_{3}$ is pointing up, and then consequently $n_{2}$ is to the left.

By inspection of Figure 4, with the velocity of $C$ being $V n_{1}$ the angular velocity of $C, \omega^{C}$ as it travels around the curve is

$$
\omega^{C}=V / R n_{3}
$$

If the train is traveling at a constant speed $V$ around the curve, $\omega^{C}$ will also be constant. Therefore the angular acceleration $\alpha^{C}$ of $C$ is

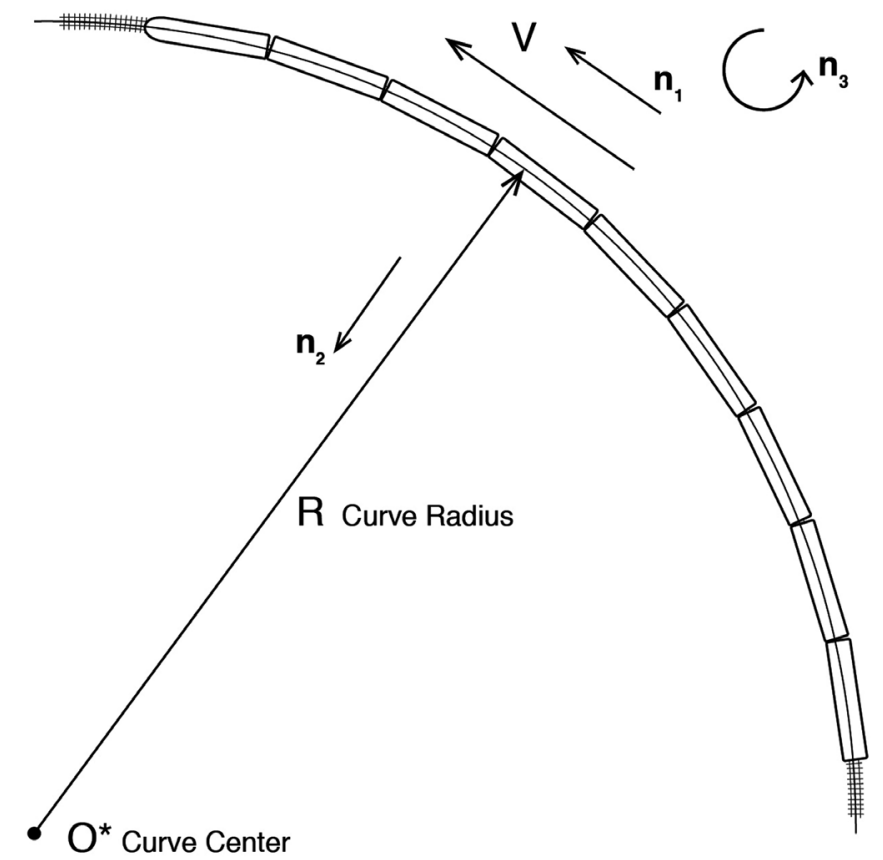

Figure 4. A high-speed train going around a curve to the left. 


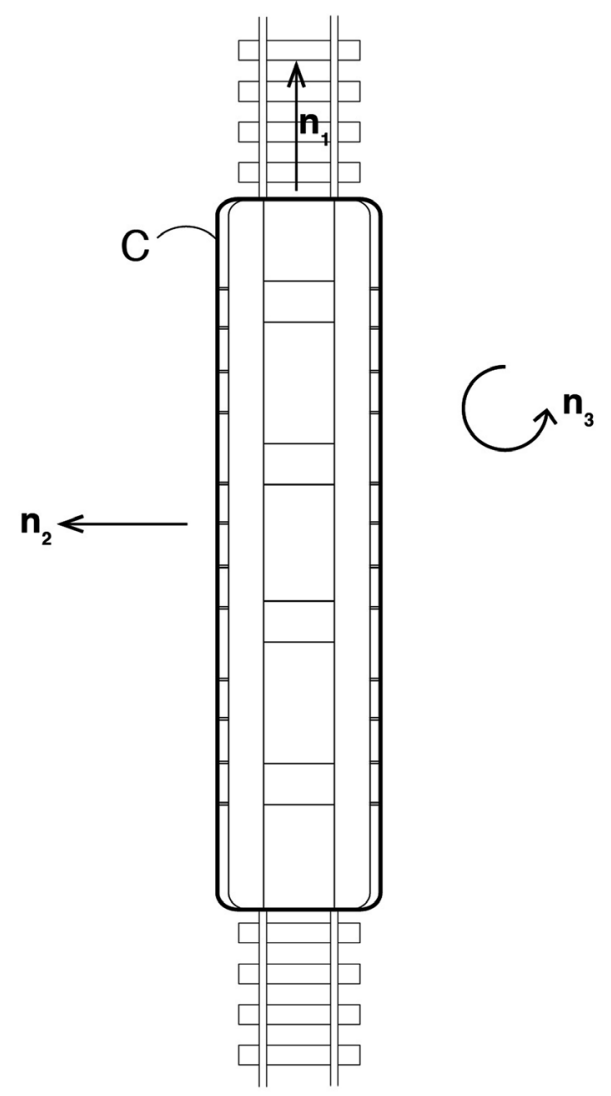

Figure 5. Overhead view of a typical rail car.

$$
\alpha^{C}=\mathrm{d} \omega^{C} / \mathrm{d} t=0
$$

Consider next a typical wheel $W$ of the rail-car as represented in Figure 6.

In Figure 6 the unit vectors provide the directions consistent with those of Figure 5. Note that in Figure 6, however, the unit vectors are not fixed relative to the wheel but instead they are fixed relative to the rail-car $C$. Consequently the angular velocity of the wheel relative to the car, written as: $\omega^{W / C}$, is seen to be:

$$
\omega^{W / C}=\Omega n_{2}=(V / r) n_{2}
$$

where $\Omega$ is the angular speed of the wheel relative to the car, and $r$ is the wheel radius (see Figure 6).

Using the addition theorem for angular velocities we see that the angular velocity of the wheel, $\omega^{W}$ in the fixed inertia frame $R^{*}$ is

$$
\omega^{W}=\omega^{W / C}+\omega^{C}=(V / r) n_{2}+(V / R) n_{3}
$$

where the last two terms are obtained by substitution from Equations (13) and (11).

Observe in Equation (14) that $\omega^{W}$ (unlike $\omega^{C}$ ) is not constant since $n_{2}$ changes direction as the train moves around the curve. Therefore, the acceleration of the wheel $\alpha^{W}$ is not zero but instead is

$$
\alpha^{W}=\mathrm{d} \omega^{W} / \mathrm{d} t=\mathrm{d}\left[(V / r) n_{2}\right] / \mathrm{d} t+\mathrm{d}\left[(V / R) n_{3}\right] / \mathrm{d} t=(V / r) \mathrm{d} n_{2} / \mathrm{d} t
$$




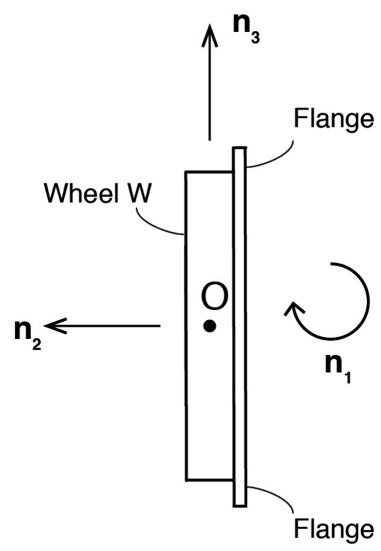

Rear View

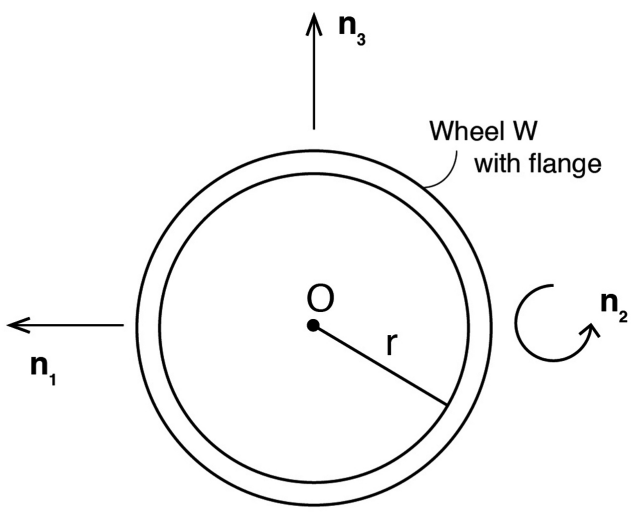

Left Side View

Figure 6. Rear and left side views of a typical rail-car wheel.

where the last term is obtained by noting that all of the parameters except $n_{2}$ are constants.

Since $n_{2}$ is fixed relative to the rail-car, its derivative is simply [3] [4] [5] [6]:

$$
\mathrm{d} n_{2} / \mathrm{d} t=\omega^{C} \times n_{2}=(V / R) n_{3} \times n_{2}=-(V / R) n_{1}
$$

Therefore, by substitution into Equation (15), $\alpha^{W}$ becomes:

$$
\alpha^{W}=-\left(V^{2} / R r\right) n_{1}
$$

Equations (14) and (17) now provide expressions for the angular velocity $\left(\omega^{W}\right)$ and the angular acceleration $\left(\alpha^{W}\right)$ of a typical wheel of the rail-car relative to the fixed inertia frame $R^{*}$. In view of Equations (8), (9), and (10) for the inertia torque components, it is convenient to use Equations (14) and (17) to obtain the $n_{i}(i=1,2,3)$ components of $\omega^{W}$ and $\alpha^{W}$. By inspection of these equations, the results are:

$$
\omega_{1}=0, \quad \omega_{2}=V / r, \quad \omega_{3}=V / R
$$

and

$$
\alpha_{1}=-V^{2} / R r, \quad \alpha_{2}=0, \quad \alpha_{3}=0
$$

\section{Rail-Car Wheel Gyroscopic Inertia Forces}

Observe that due to the circular symmetry of the wheels, the unit vectors $n_{1}$, $n_{2}$, and $n_{3}$ are parallel to principal inertia directions for the wheels, even though they are not fixed relative to the wheels.

Next, recall, or observe, from tables in References 3 to 9 that the central principal moments of inertia of a typical wheel (neglecting the flange), relative to the $n_{i}(i=1,2,3)$ unit vectors are:

$$
I_{11}=m r^{2} / 4, \quad I_{22}=m r^{2} / 2, \quad I_{33}=m r^{2} / 4
$$

where $m$ is the mass of the wheel.

Finally, by substituting from Equations (18), (19), and (20) into Equation (8) we obtain (after simplification), the $n_{i}$ components of the inertia torque as: 


$$
T_{1}=m r V^{2} / 2 R \text { and } T_{2}=T_{3}=0
$$

Observe in Equation (21) that $T_{1}$ is the only non-zero component and that $T_{1}$ is positive. With $n_{1}$ being the direction of travel around the left turning curve, we see that $T_{1}$ tends to rotate the rail-car clockwise (looking from behind). That is $T_{1}$ provides a quantification of the gyroscopic inertia torque, as described earlier via the law of gyroscopes.

\section{Rail-Car Dynamics}

Since our objective is to determine the minimum radius $R$ to keep the train cars from derailing around the curve, it is helpful to first consider the derailing tendency due to centrifugal forces. To this end, consider a rear view free-body diagram of a typical rail-car as in Figure 7.

In the figure, if derailment is to occur, the rail-car will tend to rotate about the right side rail at point $Q$. When that occurs the left side rail forces become zero. Then by setting moments about $Q$ equal to zero we obtain

$$
M g(t / 2)-\left(M V^{2} / R\right) h=0
$$

Solving Equation (22) for $V^{2}$ and $R$ we obtain

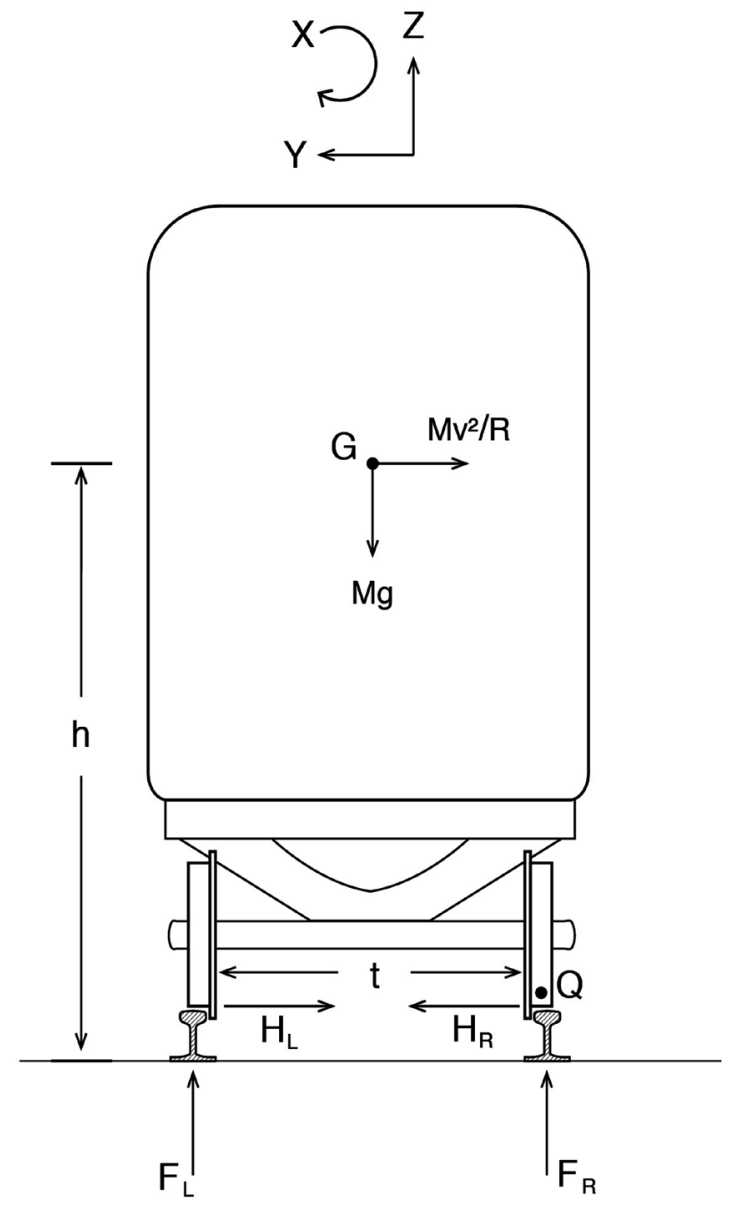

Figure 7. A simplified, rear-view, free-body diagram of a rail car. 


$$
V^{2}=R g(t \mid 2 h)
$$

and

$$
R=V^{2} \div g(t \mid 2 h)
$$

where anaaand by inspection of Figure 7, $t$ is the track width, $h$ is the height of the mass center above the rails, and $M$ is the total car mass, including the wheel masses, and $g$ is the gravity acceleration.

In Equations (23) and (24) the term: $(t / 2 h)$ is sometimes called the static stability factor [10]. The equation itself provides a simplified minimum design radius $R$ for rail-car stability around a curve. Without accounting for the gyroscopic effects of the wheels, however, the value of $R$ obtained from Equation (24) will be too small.

To account for the destabilizing effects of the wheel gyroscopic moments, consider the rear-view, free-body diagram in Figure 8. As in Figure 7, let h be the height of the mass center above the rail, and let $\mathrm{t}$ be the track width. Let $M$ be the total mass of the car including the masses of the wheels. Then by setting moments about $Q$ equal to zero, as the rail-car is about to tip off the rail, we obtain:

$$
M g(t / 2)-M\left(V^{2} / R\right) h-n m r V^{2} / 2 R=0
$$

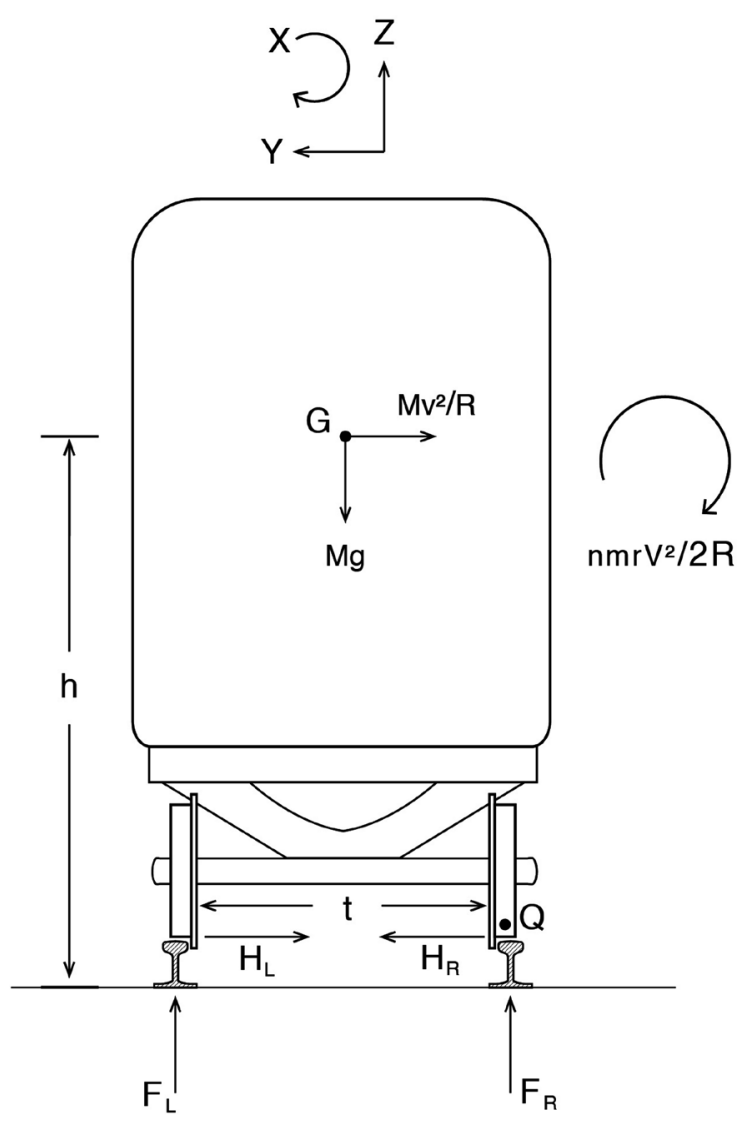

Figure 8. A rear-view, free-body diagram of a rail-car including wheel gyroscopic moments. 
where $n$ is the number of wheels on the car.

By solving for $V^{2}$ and $R$, Equation (25) takes the forms:

$$
V^{2}=R g(t \mid 2 h)[1+(m \mid M)(r \mid h)(n \mid 2)]-1
$$

and

$$
R=V^{2}[1+(M \mid m)(r \mid h)(n \mid 2)] \div g(t \mid 2 h)
$$

\section{Analysis and Discussion}

Equations (26) and (27) provide minimum design values for the speed $V$ [Equation (2)] and the curve radius $R$ [Equation (27)] for preventing rail-car derailment including both the effects of centrifugal forces and gyroscopic moment.

If the objective is to have a high-speed train, then the curve radius $R$ of Equation (27) becomes the principal design parameter.

Observe in Equation (27) there are in essence only two terms, and that the equation can be rewritten in two-term form as:

$$
R=V^{2} \div g(t / 2 h)+V^{2}(M / m)(r / h)(n / 2) \div g(t / 2 h)
$$

or as:

$$
R=R_{\text {cent }}+R_{\text {gyro }}=R_{\text {total }}
$$

where, $R_{\text {cent }}, R_{\mathrm{gyro}}$, and $R_{\text {total }}$ are defined by inspection of the two equations. $R_{\text {cent }}$ represents the portion of the curve radius required due to centrifugal forces and $R_{\text {gyro }}$ represents the portion required by the gyroscopic moments.

The question which now arises is: How significant is the term due to the gyroscopic moment of the wheels? To answer this question, consider a few typical geometric and physical values:

$$
\begin{aligned}
& V=300 \mathrm{mph}=440 \mathrm{ft} / \mathrm{sec}=483 \mathrm{~km} / \mathrm{hr}=134 \mathrm{~m} / \mathrm{s} \\
& m=34.166 \mathrm{slug}=498.5 \mathrm{~kg} \\
& M=4037.3 \mathrm{slug}=58916 \mathrm{~kg} \\
& r=1.417 \mathrm{ft}=0.4318 \mathrm{~m} \\
& h=5 \mathrm{ft}=1.524 \mathrm{~m} \\
& t=4.667 \mathrm{ft}=1.422 \mathrm{~m} \\
& g=32.2 \mathrm{ft} / \mathrm{sec}^{2}=9.8 \mathrm{~m} / \mathrm{s}^{2} \\
& n=8
\end{aligned}
$$

By substituting these values into Equations (28) and (29) we obtain:

$$
\begin{gathered}
R_{\text {cent }}=1.289 \times 104 \mathrm{ft}=3.929 \times 10^{3} \mathrm{~m} \\
R_{\text {gyro }}=1.021 \times 10^{2} \mathrm{ft}=3.112 \times 10 \mathrm{~m} \\
R_{\text {total }}=1.290 \times 10^{4} \mathrm{ft}=3.931 \times 10^{3} \mathrm{~m}
\end{gathered}
$$

Observe that the contribution $R_{\mathrm{gyro}}$ of the gyroscopic moment to the total minimum curve radius $R_{\text {total }}$ is relatively small-indeed less than $1 \%$. Even so, it is still approximately 31 meters.

As a second example, recall that with motor vehicles rollover propensity is reduced by lowering the height $h$ of the mass center above the ground. There- 
fore, let the mass center height $h$ of the rail car be reduced from $5 \mathrm{ft}(1.524 \mathrm{~m})$ to $4 \mathrm{ft}(1.219 \mathrm{~m})$, with all the other parameters remaining the same. In this case $R_{\text {cent }}, R_{\text {gyro }}$, and $R_{\text {total }}$ become:

$$
\begin{gathered}
R_{\text {cent }}=1.031 \times 10^{4} \mathrm{ft}=3.142 \times 10^{2} \mathrm{~m} \\
R_{\text {gyro }}=1.021 \times 10^{2} \mathrm{ft}=3.112 \times 10 \mathrm{~m} \\
R_{\text {total }}=1.041 \times 10^{4} \mathrm{ft}=3.453 \times 10^{2} \mathrm{~m}
\end{gathered}
$$

Observe in this case the critical curve radius is reduced due to the reduced rail-car tip over propensity. Observe also that the contribution of the gyroscopic moments to the curve length $R_{\text {gyro }}$ is unchanged since the mass center height appears in both denominators of Equation (28).

As a third example, suppose that the mass of the rail car is reduced by say $20 \%$ so that the mass is now 27.33 slug or $398.8 \mathrm{~kg}$. With all other parameters remaining the same, $R_{\text {cent }}, R_{\text {gyro }}$, and $R_{\text {total }}$ become

$$
\begin{gathered}
R_{\text {cent }}=1.289 \times 10^{4} \mathrm{ft}=3.929 \times 10^{2} \mathrm{~m} \\
R_{\text {gyro }}=1.276 \times 10^{2} \mathrm{ft}=3.889 \times 10 \mathrm{~m} \\
R_{\text {total }}=1.302 \times 10^{4} \mathrm{ft}=4.318 \times 10^{2} \mathrm{~m}
\end{gathered}
$$

Observe in this case the centrifugal tipping forces remain the same, but the effect of the wheel gyroscopic forces, which still small, is increased.

\section{Conclusions}

1) The minimum critical radius depends upon the square of the rail-car speed.

2) Equation (28) is a design formula for calculating the minimum curve radius needed to avoid tip-over derailment of a high-speed rail-car.

3) The effect of the rail-car wheel gyroscopic tip-over moment is small producing less than $1 \%$ of the minimum curve radius.

4) The lower the mass center height $h$ above the ground, the more resistant is the rail car to tip-over.

5) The mass center height does not affect the gyroscopic tip-over moment of the rail-car wheels.

6) The smaller the rail-car mass, the greater is the gyroscopic tip-over moment.

7) The rail-car mass centrifugal tip-over force is not affected by the rail car mass.

\section{Acknowledgements}

Reviewer suggestions were very helpful in preparing the final version of the paper.

\section{References}

[1] Perry, J. (1957) Spinning Tops and Gyroscopic Motion. Dover, NY.

[2] Huston, R.L., Schartman, L.S. and Connelly. J. (2013) High-Speed Motorcycle Dy- 
namics: Quick Turns While Going Straight and around Corners. International Journal of Engineering Research and Applications, 3, 1-5.

[3] Kane, T.R. (1968) Dynamics. Holt, Rinehart and Winston, NY, 91, 177.

[4] Kane, T.R. and Levinson, D.A. (1985) Dynamics, Theory and Application. McGraw Hill, NY, 125.

[5] Huston, R.L. and Liu, C.-Q. (2001) Formulas for Dynamic Analysis. Marcel Dekker Inc., New York, 149, 402-406.

[6] Josephs, H. and Huston, R.L. (2002) Dynamics of Mechanical Systems. CRC Press, Boca Raton, FL, 249-250. https://doi.org/10.1201/9781420041927

[7] Malvern, L.E. (1976) Engineering Mechanics, Vol. II Dynamics, Prentice Hall, Englewood Cliffs, NJ, 342.

[8] Beer, F.P. and Johnston Jr., E.R. (1988) Vector Mechanics for Engineers, Statics and Dynamics. 5th Edition, McGraw Hill, NY, 533.

[9] Riley, W.F. and Sturges, L.E. (1993) Engineering Mechanics, Dynamics. John Wiley \& Sons, Inc., New York, 243.

[10] Huston, R.L. (2014) Another Look at the Static Stability Factor (SSF) in Predicting Vehicle Rollover. International Journal of Crashworthiness, 18, 567-575.

Submit or recommend next manuscript to SCIRP and we will provide best service for you:

Accepting pre-submission inquiries through Email, Facebook, LinkedIn, Twitter, etc. A wide selection of journals (inclusive of 9 subjects, more than 200 journals)

Providing 24-hour high-quality service

User-friendly online submission system

Fair and swift peer-review system

Efficient typesetting and proofreading procedure

Display of the result of downloads and visits, as well as the number of cited articles

Maximum dissemination of your research work

Submit your manuscript at: http://papersubmission.scirp.org/

Or contact wjet@scirp.org 